\title{
Stable centred tetragonal phases in the hard core Yukawa system
}

\author{
Gernot J Pauschenwein ${ }^{1,2}$ and Gerhard Kahl ${ }^{1}$ \\ ${ }^{1}$ Center for Computational (CMS) Materials Science and Institut für Theoretische Physik, \\ Technische Universität Wien, Wiedner Hauptstrasse 8-10, A-1040 Wien, Austria \\ ${ }^{2}$ arsenal research, Sustainable Energy Systems, Giefinggasse 2, A-1210 Wien, Austria \\ E-mail: gernot.pauschenwein@arsenal.ac.at
}

Received 20 February 2009, in final form 21 April 2009

Published 5 November 2009

Online at stacks.iop.org/JPhysCM/21/474202

\begin{abstract}
The hard core Yukawa system, consisting of particles with an impenetrable core and interacting via a repulsive Yukawa pair potential, is known to solidify either in a bcc or fcc crystal. Using optimization strategies based on genetic algorithms we give evidence that at zero temperature an additional centred tetragonal phase structure in the range of high packing fractions emerges.
\end{abstract}

\section{Introduction}

Displacive phase transformations (such as the martensitic phase transition) are, as opposed to diffusive transformations, characterized by a cooperative, homogeneous movement of the particles in a solid crystal. This movement can be described through the so-called Bain strains, combined in a strain matrix that transforms a set of straight lines into a new set of straight lines $[1,2]$. Among the most popular and extensively studied examples of a phase transition describable through a Bain transformation is the bcc $\leftrightarrow$ fcc transition [3-5], which is, for instance, encountered in iron [6-9]. Recently computer simulations for iron [10] based on a tight-binding potential were able to reproduce this kind of phase transition.

The consideration of Bain transformations in soft matter is a comparatively young issue: recent experimental observations of bcc $\leftrightarrow$ fcc transitions in sphere-forming block copolymer/homopolymer blends were published in [11]. Another system that is able to solidify both in stable fcc as well as bcc phases and represents therefore a suitable candidate for such a transition are charged colloids. Following the Derjaguin-Landau-Verwey-Overbeek theory [12, 13], the effective interaction in a one-component colloidal dispersion of equally charged colloids in a salty solution (which guarantees the overall charge neutrality) is composed as follows: an impenetrable core which represents the finite size of the particles plus an adjacent Yukawa potential, i.e. $\Phi(r) \propto$ $\mathrm{e}^{-\kappa r} / r$. The system parameters determine the screening length $1 / \kappa$ and the prefactor of the effective two-particle potentials. In recent work it has been shown that, at high salt concentrations, many-body interactions can also be included in this simple functional form, while at low salt concentrations a truncated Yukawa potential is more appropriate to take many-body potentials into account [14-16].

The phase diagram of the hard core repulsive Yukawa system has been studied in detail (see, for instance, [17, 18]). Apart from the fluid phase, the existence of stable fcc and bcc phases has unanimously been confirmed. The phase diagram shows the dependence of the contact value of the Yukawa tail, the inverse screening length, and the packing fraction in a rich phase transition scenario. In the present contribution we give evidence that (at least) at $T=0$ a stable centred tetragonal (ct) phase can also be observed, which represents via a displacive phase transformation the bridge between the fcc and the bcc structures. It is not clear why this phase has not been identified in experiments or computer simulations to date. On the one hand this lack might be due to our observations that the phenomenon occurs only in a narrow density range and that the energy differences between competing structures are very small; another reason why this structure has not been identified before might be related to the fact that this transition can only be observed at very low temperatures.

We have identified this new and subtle feature in the phase diagram of the hard core Yukawa system using optimization tools that are based on the ideas of genetic algorithms (GAs) [19] on the one hand and the concept of metric scaling [5] on the other. GA-based search strategies have proven to be very efficient and reliable in investigations dedicated to identify ordered particle configurations of minimum energy configurations (MECs) in several soft matter systems [20-27] and to distinguish in a highly sensitive way between competing ordered structures [28]. In the present 
context, the GA-based search strategy was able to identify the existence of a stable centred tetragonal structure. The metric scaling concept [5], on the other hand, has been used to locate the transition densities with high accuracy.

This paper is organized as follows: we start by introducing the model and our theoretical tools. In section 3 we present and discuss our results and close the paper with concluding remarks.

\section{Model and theoretical tools}

\subsection{Model}

The pair potential in the (repulsive) hard core Yukawa model is given by

$$
\Phi(r)= \begin{cases}\infty & r<\sigma \\ \frac{\epsilon \sigma}{r} \mathrm{e}^{-\kappa(r-\sigma)} & r \geqslant \sigma,\end{cases}
$$

where $\sigma$ is the hard core diameter, $\epsilon$ is the energy at contact and $\kappa$ is the range parameter (or inverse screening length). $\sigma$ and $\epsilon$ are our natural length and energy units; related dimensionless quantities will be indicated by a star superscript, i.e. $r^{\star}=r / \sigma$ and $\kappa^{\star}=\kappa \sigma$. We further introduce the number density $\rho$ (or its dimensionless counterpart, $\rho^{\star}=\rho \sigma^{3}$ ) and the packing fraction $\eta=\rho^{\star} \pi / 6$.

Our investigations are performed in the NPT ensemble since a proper inclusion of the hard core in the GA framework can be formulated more conveniently in this ensemble [26, 27]. Under these conditions, the equilibrium structures are identified by minimizing the Gibbs free energy, $G$; we introduce at this point the Gibbs free energy per particle, $g=G / N$, and a further closely related, dimensionless quantity, $g^{\star}=g / \epsilon$. To be consistent with previous contributions we term the configurations that minimize the Gibbs free energy as MECs.

At zero temperature, $g=e+P / \rho$. Here $P$ is the pressure (or, in its reduced dimensionless form, $P^{\star}=P \sigma^{3} / \epsilon$ ), $\rho$ is the particle density and $e$ is the lattice sum per particle and per energy unit, i.e. $e^{\star}=E /(N \epsilon), E$ being the lattice sum. For the reduced quantities we obtain the following relation:

$$
g^{\star}=e^{\star}+\frac{P^{\star}}{\rho^{\star}}
$$

Due to the infinite range of the Yukawa tail, summations have to be truncated at a cutoff radius $r_{\text {cut }}$, guaranteeing $\Phi(r)$ to be sufficiently small for $r>r_{\text {cut }}$. To be more specific, $r_{\text {cut }}$ is defined in the present contribution via

$$
\delta \int_{\sigma}^{\infty} \mathrm{d}^{3} r \Phi(r)=\int_{r_{\text {cut }}}^{\infty} \mathrm{d}^{3} r \Phi(r),
$$

a criterion which guarantees that the neglected contributions to the lattice sums are of the order of $\delta$ times the total energy; in our calculations we used $\delta=10^{-10}$.

\subsection{Theoretical tools}

To calculate the phase diagram at $T=0$ a set of candidate crystal structures is needed. We obtained these structures via our search strategy based on GAs. This approach delivers in our ensemble the equilibrium density $\rho$ and provides with a high success rate the equilibrium crystal structure $\mathcal{C}$ at given pressure $P$, i.e. the three vectors defining the unit cell and the positions of the additional basis particles. For technical details we refer to [27] and references therein. Applying the GA-based search strategy for our particular system at $T=0$, has led to the fcc, the ct and the bcc structures as possible candidates.

In order to draw the full phase diagram at $T=0$, we have to identify the transition pressures. For the case of the square shoulder, this task was reasonably easy due to the fact that for this particular system $g^{\star}$ is a linear function of the pressure (cf discussions in $[26,27]$ ) and transition points can easily and exactly be determined by intersecting straight lines. For the HCY system, however, $g^{\star}$ is no longer a linear function of $P^{\star}$; thus, in an effort to locate the transition pressure values of competing structures with high accuracy we have taken recourse to the concept of metric scaling presented in [5] and specified in the following.

We start with the candidate structures of cubic symmetry (i.e. bcc and fcc lattices) which are characterized by a single free parameter, i.e. the edge length of the cubic box. We introduce a type of scaling, which is characterized by a single scaling factor $s$, that scales all distances and hence preserves symmetry. For convenience we define the reference structure as the one where the cores of the particles are in direct contact and set $s=1$ for this close-packed structure. Obviously this value of $s$ represents the smallest possible $s$ value, which we denote as $s_{\text {low }}$. Let us consider a crystal lattice of cubic symmetry, $\mathcal{C}_{0}$, and of density $\rho_{0}$, which represents the MEC at $P_{0}$ and which, in addition, is characterized by a scaling parameter $s_{0}$. Then we denote a structure that is obtained by scaling $\mathcal{C}_{0}$, with the factor $s$ as $\tilde{\mathcal{C}}\left(\mathcal{C}_{0}, s\right)$. We now consider the class of structures $\left\{\tilde{\mathcal{C}}\left(\mathcal{C}_{0}, s\right) \mid s\right.$ positive $\}$ : among these scaled structures we look for the one with the lowest Gibbs free energy per particle at given pressure; this value of $s$ is termed $\bar{s}$ and the corresponding thermodynamic potentials is denoted as $g\left(P ; \mathcal{C}_{0}, \bar{s}\right)$.

In the following we give evidence that, for the cubic structures, $\bar{s}$ is uniquely defined. Let us consider for the moment only the Yukawa contribution to the potential (1), i.e. ignoring the hard core. Since the Yukawa tail is positive for all $r$ and all $\kappa$, we obtain for obvious reasons

$$
\begin{array}{cc}
e\left(\mathcal{C}_{0}, s\right)>0, & \frac{\partial e\left(\mathcal{C}_{0}, s\right)}{\partial s}<0, \\
\text { and } & \frac{\partial^{2} e\left(\mathcal{C}_{0}, s\right)}{\partial s^{2}}>0
\end{array}
$$

for all $s>0$, where $e\left(\mathcal{C}_{0}, s\right)$ stands for the lattice sum per particle of the crystal structure obtained by scaling $\mathcal{C}_{0}$ with $s$. In addition, since the equilibrium density $\rho_{0}$ of $\mathcal{C}_{0}$ is also known, the density of the scaled crystal is simply given by $\rho(s)=\left(\rho_{0} s_{0}^{3}\right) / s^{3}$. Hence, the pressure contribution to the 


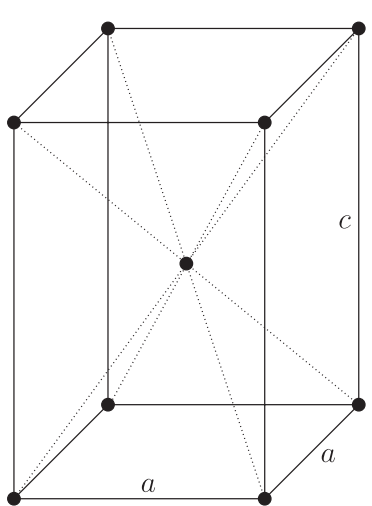

Figure 1. Sketch of the (body) centred tetragonal lattice, with edge lengths $a, a$ and $c$ as labelled.

Gibbs free energy per particle is $P s^{3} /\left(\rho_{0} s_{0}^{3}\right)$ and we obtain in total

$$
g\left(P ; \mathcal{C}_{0}, s\right)=e\left(\mathcal{C}_{0}, s\right)+P s^{3} /\left(\rho_{0} s_{0}^{3}\right) .
$$

From equations (4) and (5) it is obvious that

$$
\frac{\partial^{2} g\left(P ; \mathcal{C}_{0}, s\right)}{\partial s^{2}}>0 \quad \text { for all } P \text { and } s>0 .
$$

For $s \rightarrow 0, g\left(P ; \mathcal{C}_{0}, s\right)$ becomes infinite due to the $1 / r$ repulsion; for $s \rightarrow \infty, g\left(P ; \mathcal{C}_{0}, s\right)$ becomes infinite due to the second contribution in equation (5), i.e.

$$
\lim _{s \rightarrow 0} g\left(P ; \mathcal{C}_{0}, s\right)=\infty \quad \text { and } \quad \lim _{s \rightarrow \infty} g\left(P ; \mathcal{C}_{0}, s\right)=\infty
$$

These two limiting cases of $g\left(P ; \mathcal{C}_{0}, s\right)$ in combination with the fact that this function is convex for positive $s$ values (cf equation (6)) guarantees that there exists exactly one minimum of $g\left(P, \mathcal{C}_{0}, s\right)$ for $s>0$, which we denote as $s_{\min }$.

Taking the hard core and the cutoff radius into account now, it is obvious that there exists (i) a smallest possible scaling factor, $s_{\text {low }}=1$ (introduced above), as well as (ii) a largest possible scaling factor of $s_{\text {high, where the shortest }}$ distance between two points in the entire lattice is equal to $r_{\text {cut }}$. Therefore, the equilibrium structure out of the set of scaled structures $\left\{\tilde{\mathcal{C}}\left(\mathcal{C}_{0}, s\right) \mid s\right.$ positive $\}$ is obtained by a scaling factor, $s_{\text {eq }}$, which is in general $s_{\text {min }}$. Only if $s_{\text {min }}<s_{\text {low }}$ is $s_{\text {eq }}=s_{\text {low }}$ and in the case $s_{\min }>s_{\text {high }}$ then $s_{\text {eq }}=s_{\text {high }}$. With all this in mind, $g(P)$ can be determined - starting from the value $P_{0}$ for any $P$ value.

Extending the concept of metric scaling to the ct structure we have to include an additional metric scaling parameter, $f$. The ct lattice, visualized in figure 1 , is characterized by edge lengths $a, a$ and $c$.

For reasons explained below we use the following scaling relations:

$$
c(f, s)= \begin{cases}s \sigma & f<\frac{\sqrt{3}}{2} \\ s f \frac{2 \sigma}{\sqrt{3}} & \frac{\sqrt{3}}{2} \leqslant f\end{cases}
$$

and

$$
a(f, s)= \begin{cases}s \frac{3 \sigma}{2 \sqrt{2}} \frac{1}{f} & f<\frac{\sqrt{3}}{2} \\ s \sqrt{3-f^{2}} \sqrt{\frac{2}{3}} \sigma & \frac{\sqrt{3}}{2} \leqslant f \leqslant \sqrt{\frac{3}{2}} \\ s \sigma & \sqrt{\frac{3}{2}}<f .\end{cases}
$$

While $s$ scales all distances to the same amount, $f$ guarantees that the nearest-neighbour distance between the particles in the corresponding ct structure is kept constant. The above definitions for $c(f, s)$ and $a(f, s)$ impose in the interval $\sqrt{3} / 2 \leqslant f \leqslant \sqrt{3 / 2}$ that (i) the value of the tridiagonal (volume diagonal) is kept to a constant value of $2 \sigma s$ and that (ii) the reduced density, $\rho^{\star}$, varies with $s$ and $f$ according to

$$
\rho^{\star}=\frac{1}{s^{3}} \frac{3 \sqrt{3}}{6 f-2 f^{3}} .
$$

In the scaling relations (8) and (9) the case $f<\sqrt{3} / 2$ corresponds to the situation where $c$ represents the shortest edge of the conventional unit cell, while the case $f \geqslant \sqrt{3 / 2}$ corresponds to $a$ being the shortest edge of the cell. In the intermediate $f$ range, i.e. for $\sqrt{3} / 2 \leqslant f \leqslant \sqrt{3 / 2}$, the above scaling realizes a Bain transformation from a bcc (with $f=1$ ) to an fcc (with $f=\sqrt{3 / 2}$ ) lattice. Furthermore, we denote bcc and fcc lattices where particles are in direct contact (i.e. for $s=1$ ) by $\mathrm{bcc}_{\sigma}$ and $\mathrm{fcc}_{\sigma}$, respectively. In figure 2 the Bain transformation from $\mathrm{bcc}_{\sigma}$ to $\mathrm{fcc}_{\sigma}$ via the $\mathrm{ct}_{\sigma}$ structure(s) is visualized: while $s$ is kept constant at $1, f$ varies from 1 to $\sqrt{3 / 2}$.

In an effort to obtain the MECs formed by the ct lattice as functions of $s$ and $f$, we proceed similarly as in the previous case: assume we have a crystal lattice $\mathcal{C}_{0}$, which represents the MEC at $P_{0}$ and has density $\rho_{0}$, and which, in addition, is characterized by scaling parameters $s_{0}$ and $f_{0}$. Then we denote a structure that is obtained by scaling $\mathcal{C}_{0}$, with the factors $s$ and $f$ as $\tilde{\mathcal{C}}\left(\mathcal{C}_{0}, s, f\right)$. If we now consider the class of structures $\left\{\tilde{\mathcal{C}}\left(\mathcal{C}_{0}, s, f\right) \mid s\right.$ and $f$ positive $\}$, then we search the values of $s$ and $f$ (termed $\bar{s}$ and $\bar{f})$, so that $\tilde{\mathcal{C}}\left(\mathcal{C}_{0}, \bar{s}, \bar{f}\right)$ minimizes the Gibbs free energy per particle at some given pressure $P$, which we denote as $g\left(P ; \mathcal{C}_{0}, \bar{s}, \bar{f}\right)$. In contrast to the lattices with cubic symmetry, the ct-based structures have to be identified via numerical minimization.

\section{Results}

Working in the NPT ensemble, we impose a pressure value, $P^{\star}$, and select a $\kappa$ value; as a result the optimization procedure yields the equilibrium density for this particular state point. A first search for equilibrium structures on a coarse $\left(P^{\star}, \kappa^{\star}\right)$ grid using GA-based optimization techniques indicates the emergence of three equilibrium structures: bcc, fcc and ct lattices. The exact location of the stability ranges and the transition pressures between the competing structures has been realized with the concept of metric scaling as outlined in section 2.2. 


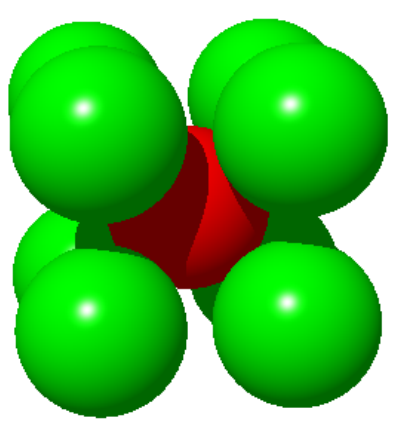

(a)

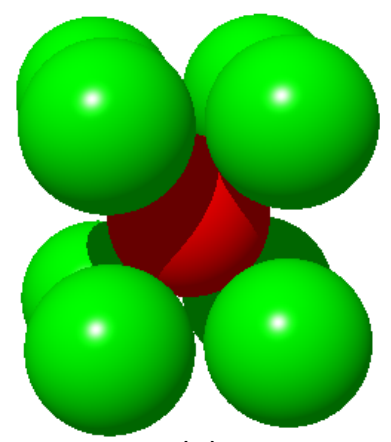

(c)

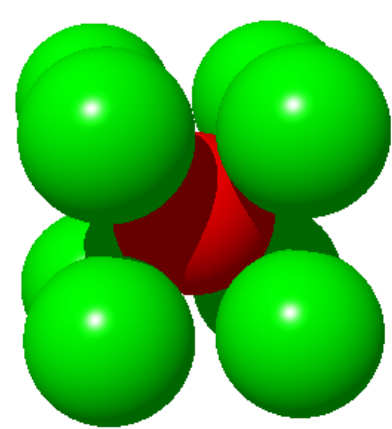

(b)

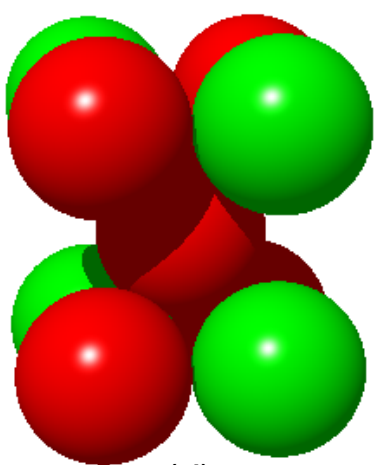

(d)
Figure 2. Visualization of the Bain transformation via variation of $f$ at constant $s=1$ from the $\mathrm{bcc}_{\sigma}$ to the $\mathrm{fcc}_{\sigma}$ structure via two intermediate $\mathrm{ct}_{\sigma}$ structures as labelled. In (a)-(c) the centred particle is the one with the dark shading (red), while in (d) a side face of the conventional $\mathrm{fcc}_{\sigma}$ unit cell is marked by the particles with darker shading (red). (a) $f=1\left(\right.$ bcc $\left._{\sigma}\right)$, (b) $f=1.05$ ( $\left.\mathrm{ct}_{\sigma}\right)$, (c) $f=1.15\left(\mathrm{ct}_{\sigma}\right)$ and (d) $f=\sqrt{3 / 2}\left(\mathrm{fcc}_{\sigma}\right)$.

(This figure is in colour only in the electronic version)

Anticipating that the ct structure is encountered only in a narrow region of parameter space, we start with the presentation of the zero temperature phase diagram where only the bcc and fcc structures are included: they are depicted in figure 3(a) in the $\left(\kappa^{\star}, P^{\star}\right)$ plane and in figure 4(a) in the $\left(\kappa^{\star}, \eta\right)$ plane. From figure 3(a) we learn that at zero temperature and for $\kappa^{\star}$ values up to $\kappa_{\max }^{\star} \simeq 1.875$ three phases are observed: at low pressure values, an fcc lattice is stable, which transforms upon compression via a first-order phase transition into a bcc lattice; then follows the ct phase (to be discussed below) which then transforms into an fcc phase and for higher pressure values into an $\mathrm{fcc}_{\sigma}$ phase, which remains stable up to arbitrarily high pressure. The $\kappa^{\star}$ range where the bcc phase is stable extends from $\kappa=0$ to $\kappa_{\max }^{\star}$. Along the high pressure solid line a $\mathrm{bcc}_{\sigma}$ phase is stable (cf the label in 3(a)) where the hard cores of the particles are in direct contact, thus $\eta_{\text {bcc }_{\sigma}}=\pi \sqrt{3} / 8 \sim 0.6802$. The range where the bcc phase is stable is indicated in figure 3(a) by a solid line. For $\kappa \gtrsim \kappa_{\max }^{\star}$ all equilibrium structures are fcc crystals: among these the cores of the particles are above a $\kappa$-dependent pressure threshold, i.e. $P^{\star}=P_{\mathrm{fcc}_{\sigma}}^{\star}\left(\kappa^{\star}\right)$, in direct contact, forming thus $\mathrm{fcc}_{\sigma}$ structures. The dashed line in figure 3(a) indicates the limiting pressure above which the system forms an $\mathrm{fcc}_{\sigma}$ structure. Finally, in the region between the full and
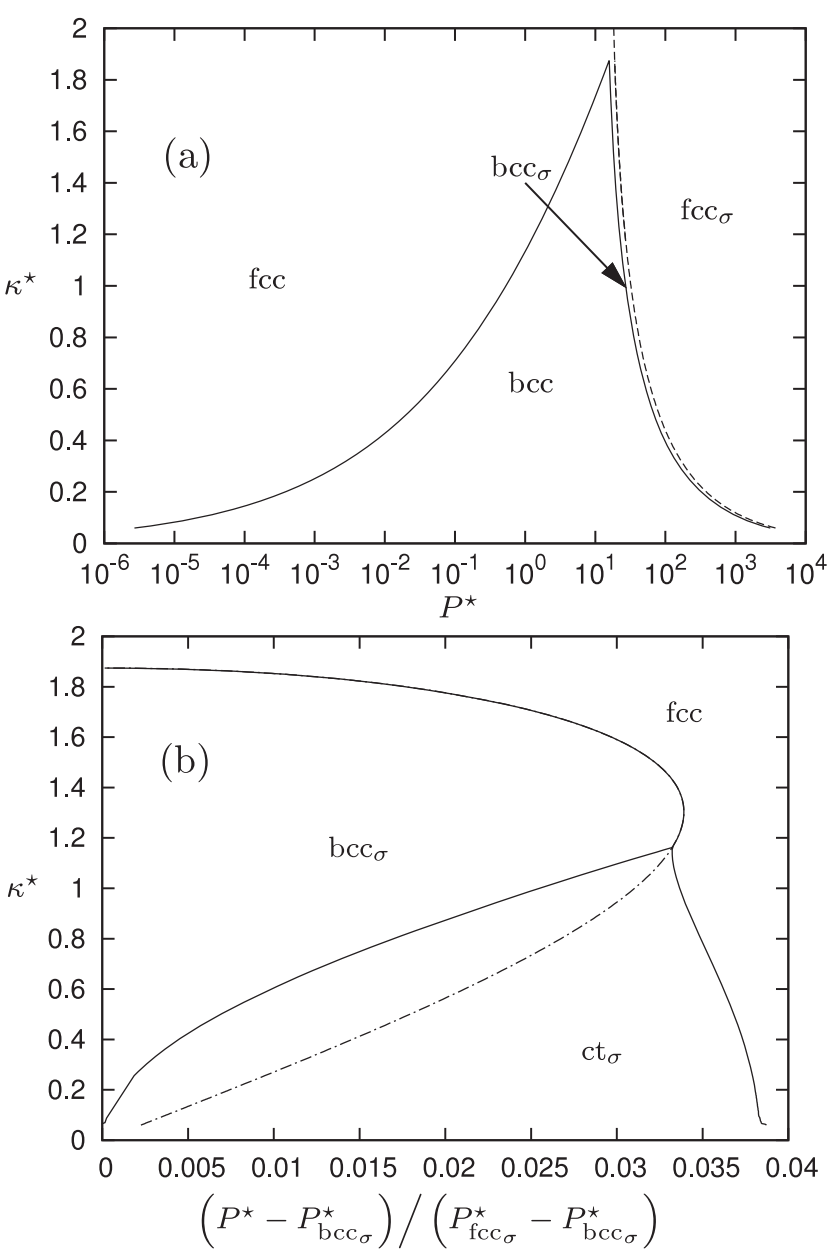

Figure 3. (a) A zero temperature phase diagram for the hard core Yukawa model in the $\left(\kappa^{\star}, P^{\star}\right)$ plane involving only the cubic phases. Solid lines delimit the stability range of the bcc phase, while the dashed line indicates the limiting pressure above which the particles form a close-packed fcc $\sigma$ structure. (b) An enlarged region of the zero temperature phase diagram of the hard core Yukawa model in the $\left(\kappa^{\star}, P^{\star}\right)$ range where the $\mathrm{ct}_{\sigma}$ lattice represents the energetically most favourable structure. The dashed-dotted line indicates the transition line between the $\mathrm{bcc}_{\sigma}$ and fcc structures if only cubic lattices were considered. In these pressure units the $\mathrm{fcc}_{\sigma}$ phase is only encountered outside the displayed range.

the dashed lines, the ct phase is stable: this part of the phase diagram will be discussed below.

In figure 4 the zero temperature phase diagram for our system is depicted in the $\left(\kappa^{\star}, \eta\right)$ plane. We see that the bcc phase is encountered in particular in the low- $\kappa$ range; it remains stable for packing fractions up to $\eta=\pi \sqrt{3} / 8$, where a $\mathrm{bcc}_{\sigma}$ structure is formed. Then, upon further increasing the density a ct phase emerges in a narrow $\eta$ range (to be discussed below), which then transforms into an fcc lattice. Finally, at $\eta=\pi / 3 \sqrt{2}$ an $\mathrm{fcc}_{\sigma}$ phase is formed where the cores are in direct contact and no further compression is possible. Outside a parabola-shaped area, i.e. at intermediate and high $\kappa$ values, only the fcc phase is stable. We have complemented our data with the phase diagram of the hard core Yukawa system for $T>0$, taking data from [18]. Of course, at finite temperatures a fluid phase also emerges at low densities. As one can see 

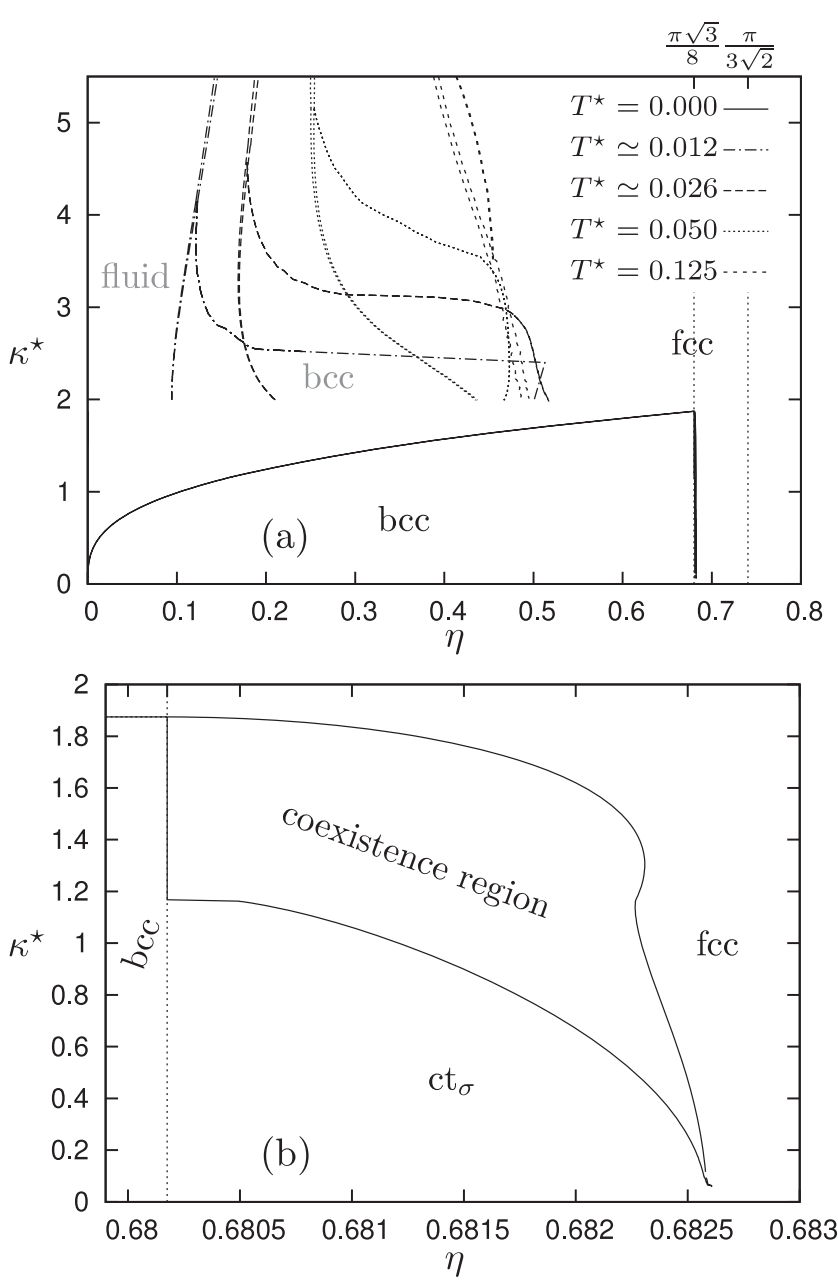

Figure 4. (a) A phase diagram for the hard core Yukawa model in the $\left(\kappa^{\star}, \eta\right)$ plane involving only the cubic phases and, for $T>0$, the fluid phases (as labelled); temperatures as indicated in the inset. Data for $T>0$ have been taken from [18]. Vertical broken lines indicate the packing fractions of bcc $_{\sigma}$ and fcc $\sigma$ phases, i.e. $\eta=\pi \sqrt{3} / 8$ and $\eta=\pi / 3 \sqrt{2}$, respectively. (b) An enlarged region of the zero temperature phase diagram of the hard core Yukawa model in the $\left(\kappa^{\star}, \eta\right)$ range where the $\mathrm{ct}_{\sigma}$ lattice represents the energetically most favourable structure.

in figure 4(a), the range where the bcc structure is stable nicely merges with vanishing temperature with our $(T=0)$ prediction of the coexistence curve.

Now we turn to the identification of the ct phase, emerging in a narrow $\eta$ interval between bcc $_{\sigma}$ and the fcc phase. In an effort to locate these transitions, we intersect the $g$ curves, evaluated for the competing structures over representative $P$ ranges. While for the cubic phases minimization of $g$ with respect to the only scaling parameter $s$ is rather trivial, the determination of the corresponding curve for the ct structure is more delicate since optimization has to be done with respect to the two scaling parameters $s$ and $f$. However, in our investigations we have observed the following: in the pressure range where the ct structure is found to be the most stable one, we always find $s=1$, corresponding thus to a $\mathrm{ct}_{\sigma}$ structure where the cores of the particles are always in direct contact. Once the $g^{\star}(P)$ curves for all three competing structures have

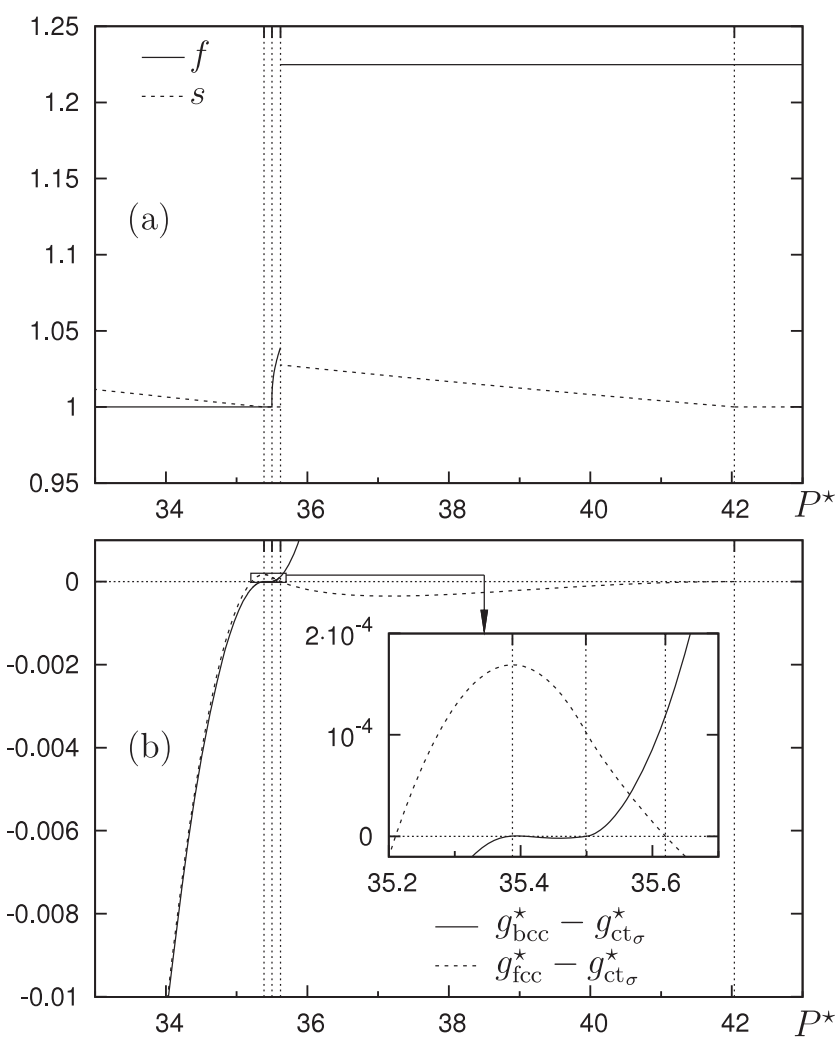

Figure 5. (a) Scaling parameters $s$ and $f$ as introduced in the text (see section 2.2), characterizing the ordered equilibrium structures for the hard core Yukawa system for $\kappa^{\star}=0.4$ as functions of the reduced pressure $P^{\star}$ as labelled. (b) Differences in the reduced Gibbs free energy, $g_{\mathrm{bcc}^{\star}}^{\star}-g_{\mathrm{ct}_{\sigma}}^{\star}$ and $g_{\mathrm{fcc}}^{\star}-g_{\mathrm{ct}_{\sigma}}^{\star}$, of the competing structures as labelled as functions of the reduced pressure $P^{\star}$. Vertical dotted lines separate (from left to right) regions where the bcc, bcc $\sigma, \mathrm{ct}_{\sigma}, \mathrm{fcc}$ and $\mathrm{fcc}_{\sigma}$ structures represent the energetically most favourable lattices. The inset covers those pressure ranges where the ct lattice is the most stable structure.

been evaluated, we determine numerically their intersection points which define the transition pressure values.

Now we are able to trace the full phase diagram. Enlarged views of the relevant parts are depicted in figure 3(b) in the $\left(\kappa^{\star}, P^{\star}\right)$ plane and in figure $4(\mathrm{~b})$ in the $\left(\kappa^{\star}, \eta\right)$ plane. In the former case we have used a rescaled pressure axis to guarantee an optimum visibility of the range of stability of the respective phases: here, $P_{\mathrm{bcc}_{\sigma}}^{\star}$ and $P_{\mathrm{fcc}_{\sigma}}^{\star}$ represent the smallest pressure values for which the $\mathrm{bcc}_{\sigma}$ and $\mathrm{fcc}_{\sigma}$ phases are stable, respectively; we note that both $P_{\mathrm{bcc}_{\sigma}}^{\star}$ and $P_{\mathrm{fcc}_{\sigma}}^{\star}$ are functions of $\kappa^{\star}$, whose actual values can be inferred from figure 3(a). From figure 3(b) we learn that for $1.16 \lesssim \kappa^{\star} \lesssim 1.875$ a direct transition from the $\mathrm{bcc}_{\sigma}$ to the fcc lattice is observed, while for $\kappa^{\star} \lesssim 1.16$ an intermediate $\mathrm{ct}_{\sigma}$ phase is the energetically most favourable one. The region in the $\left(\kappa^{\star}, \eta\right)$ plane where the $\mathrm{ct}_{\sigma}$ structure is stable is visualized in figure 4(b).

In figures 5 and 6 , we display the scaling parameters $s$ and $f$ as well as the Gibbs free energy as functions of $P^{\star}$ considering two different $\kappa^{\star}$ values: $\kappa^{\star}=0.4$ and 1.4. In the first case, the $\mathrm{ct}_{\sigma}$ structure is stable in a narrow pressure range, while at $\kappa^{\star}=1.4$ a direct transition from the bcc $_{\sigma}$ to the fcc structure occurs. From the pressure dependence of the scaling 


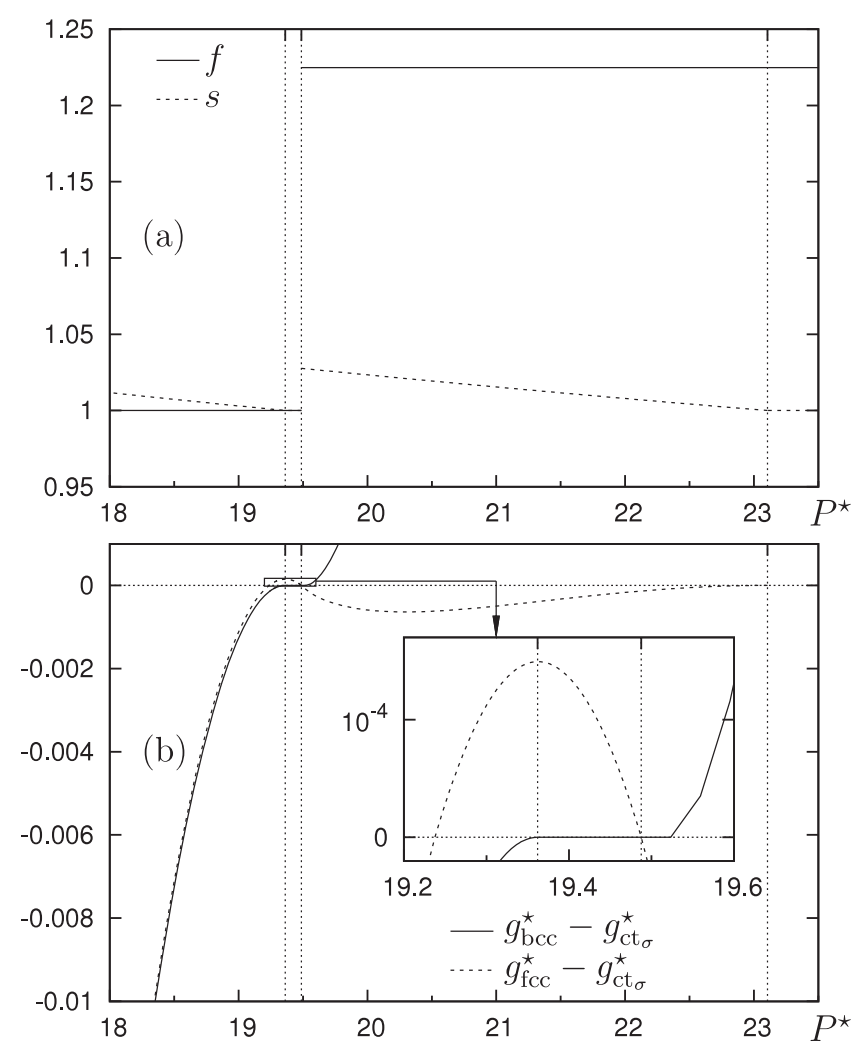

Figure 6. Same as figure 5 for $\kappa^{\star}=1.4$. Note that for this $\kappa^{\star}$ value and over the entire pressure range the ct structure is energetically less favourable than the bcc or the fcc lattices.

parameters we can nicely trace the respective transitions: starting for $\kappa^{\star}=0.4$, we first observe at low pressure values a bcc structure $(s>1)$ which transforms into a bcc $\sigma$ structure $(s=1)$. Then a ct ${ }_{\sigma}$ structure occurs (i.e. $\left.s \sim 1\right)$, which first transforms into an fcc lattice and under higher pressure becomes an $\mathrm{fcc}_{\sigma}$ structure. Concomitantly, $f$ keeps the value of 1 for the bcc and the $\mathrm{bcc}_{\sigma}$ phases and then continuously increases for the $\mathrm{ct}_{\sigma}$ phase to a limiting value of $f \sim 1.05$ (for $\left.\kappa^{\star}=0.4\right)$. Then $f$ changes discontinuously to $f=\sqrt{3 / 2}$, i.e. the value that characterizes the fcc structure. We conclude from these observations that the transition $\mathrm{bcc}_{\sigma} \rightarrow \mathrm{ct}_{\sigma}$ is continuous, while the change from $\mathrm{ct}_{\sigma} \rightarrow$ fcc is (most likely) discontinuous. For $\kappa^{\star}=1.4$ where no ct structure appears, $f$ changes discontinuously from $f=1$ (bcc) to $f=\sqrt{3 / 2}$ (fcc). Again, the $s$ curves provide information whether particles are in the respective lattices in direct contact (i.e. $s=1$ ) or not (i.e. $s>1$ ). The energy curves (depicted in the respective (b) panels of figures 5 and 6) give evidence that the differences between all competing structures are very small.

\section{Conclusion}

We have successfully applied our GA-based search strategy to identify the zero temperature phase diagram of the hard core Yukawa model and have revealed the existence of stable ct structures at very high pressure values, as long as the range parameter $\kappa$ is not too large. The region in the phase diagram where the ct structures turns out to be stable is found to be rather narrow and the energy differences between the competing structures are very small. These two facts are most likely the reason why the ct structure has not been identified for the hard core Yukawa system, yet: computer simulations and other theoretical approaches (that are based on approximate schemes) have problems in providing results for thermodynamic potentials of sufficient accuracy and in experiments it might be difficult to exactly identify the narrow parameter range where a ct structure should be stable. On the other hand, our investigations impressively demonstrate that GA-based optimization techniques are able to distinguish between competing lattices even if the differences in their respective energies are very small.

\section{Acknowledgments}

The authors are indebted to Julia Fornleitner and Dieter Gottwald (both Wien) for stimulating discussions and for computational aid. Financial support by the Austrian Science Foundation (FWF) under project nos. W004, P17823-N08 and P19890-N16 is gratefully acknowledged. The authors are indebted to Antti-Pekka Hynninen and Marjolein Dijkstra for providing data published in [18] and presented in figure 4(a).

\section{References}

[1] Bain E C 1924 Trans. AIME 7025

[2] Nishiyama Z, Fine M E, Meshii M and Wayman C M 1978 Martensitic Transformation (New York: Academic)

[3] Beauchamp P and Villain J P 1983 J. Physique 441117

[4] Milstein F, Fang H E and Marschall J 1994 Phil. Mag. 70621

[5] Miller M A and Reinhardt W P 2000 J. Chem. Phys. 1137035

[6] Olsen G H and Jesser W A 1971 Acta Metall. Mater. 191009

[7] Olsen G H and Jesser W A 1971 Acta Metall. Mater. 191299

[8] Cuenya B R, Doi M, Löbus S, Courths R and Keune W 2001 Surf. Sci. 493338

[9] Suzuki T, Shimono M and Kajiwara S 2001 Mater. Sci. Eng. A 312104

[10] Engin C and Urbassek H M 2008 Comput. Mater. Sci. 41297

[11] Huang Y-Y, Hsu J-Y, Chen H L and Hashimoto T 2007 Macromolecules $\mathbf{4 0} 3700$

[12] Derjaguin B V and Landau L 1941 Acta Phys.-Chim. 14633

[13] Verwey E J W, Overbeek J T G and van Nes K 1948 Theory of the Stability of Lyophobic Colloids (New York: Elsevier)

[14] Dobnikar J, Chen Y, Rzehak R and von Grünberg H H 2003 J. Phys.: Condens. Matter 15 S263

[15] Dobnikar J, Rzehak R and von Grünberg H H 2003 Europhys. Lett. 61695

[16] Dobnikar J, Chen Y, Rzehak R and von Grünberg H H 2003 J. Chem. Phys. 1194971

[17] Meijer E J and El Azhar F 1997 J. Chem. Phys. 1064678

El Azhar F, Baus M, Ryckaert J-P and Meijer E J 2000 J. Chem. Phys. 1125121

[18] Hynninen A-P and Dijkstra M 2003 Phys. Rev. E 68021407

[19] Holland J 1975 Adaption in Natural and Artificial Systems (Ann Arbor, MI: University of Michigan Press)

[20] Gottwald D, Kahl G and Likos C N 2005 J. Chem. Phys. 122204503

[21] Mladek B M, Gottwald D, Kahl G, Neumann M and Likos C N 2006 Phys. Rev. Lett. 96045701 
Mladek B M, Gottwald D, Kahl G, Neumann M and Likos C N 2006 Phys. Rev. Lett. 97019901

[22] Mladek B M, Gottwald D, Kahl G, Likos C N and Neumann M 2007 J. Phys. Chem. B 11112799

[23] Fornleitner J and Kahl G 2008 Europhys. Lett. 8218001

[24] Fornleitner J, Lo Verso F, Kahl G and Likos C N 2008 Soft Matter 4480
[25] Fornleitner J, Lo Verso F, Kahl G and Likos C N 2009 Langmuir at press (doi:10.1021/1a900421v)

[26] Pauschenwein G J and Kahl G 2008 Soft Matter 41396

[27] Pauschenwein G J and Kahl G 2008 J. Chem. Phys. 129174107

[28] Kahn M, Weis J-J, Likos C N and Kahl G 2009 Soft Matter at press 